\title{
Challenges in the management of juvenile idiopathic arthritis with etanercept
}

\author{
Clare E Pain \\ Liza J McCann
}

Alder Hey Children's NHS Foundation

Trust, Eaton Road, Liverpool, UK
Correspondence: Liza McCann

Consultant Paediatric Rheumatologist, Alder Hey Children's NHS Foundation Trust, Eaton Road, Liverpool, LI2 2AP, UK $\mathrm{Tel}+4401512525153$

Fax +44 0I5I 2525928

Email liza.mccann@alderhey.nhs.uk

\begin{abstract}
Biologic agents have been designed with the help of immunological studies to target particular areas of the immune system which are thought to play a role in the pathogenesis of disease. Etanercept is a soluble anti-tumor necrosis factor alpha (TNF- $\alpha$ ) agent licensed for the treatment of active poly-articular juvenile idiopathic arthritis (JIA) in children aged 4 to 17 years who have failed to respond to methotrexate alone, or who have been intolerant of methotrexate. The safety and efficacy of etanercept in this patient group has been established by one randomized controlled trial and several longitudinal studies. This, together with the fact that until recently etanercept was the only anti-TNF licensed in JIA, has made it the most common first choice biologic for many clinicians. However, there are still many unanswered questions about etanercept, including its efficacy and safety in different subtypes of JIA, in children under 4 years of age and in those with uveitis. There are still concerns about the long term safety of TNF antagonists in the pediatric age group and unanswered questions about increased risks of malignancy and infection. Although adult studies are useful to improve understanding of these risks, they are not a substitute for good quality pediatric research and follow-up studies. Adult trials often include greater numbers of patients. However, they evaluate a different population and drug behavior may vary in children due to differences in metabolism, growth and impact on a developing immune system. In addition, rheumatoid arthritis is a different disease than JIA. Clinicians need to carefully weigh up the risk benefit ratio of anti-TNF use in children with JIA and push for robust clinical trials to address the questions that remain unanswered. This article summarizes the evidence available for use of etanercept in children with JIA and highlights aspects of treatment in need of further research.
\end{abstract}

Keywords: juvenile idiopathic arthritis, biologic therapy, etanercept

\section{Introduction}

Juvenile idiopathic arthritis (JIA) is a heterogeneous group of conditions defined as inflammatory arthritis occurring in those under 16 years of age, present for greater than 6 weeks, after exclusion of other causes. The revised International League Against Rheumatism (ILAR) classification defines 7 subsets of JIA (Table 1). ${ }^{1}$ Management and treatment response differ between subgroups. Many children respond to traditional treatments which include a combination of non-steroidal anti-inflammatory drugs (NSAIDs), corticosteroids (intra-articular, pulsed intravenous, oral) and disease modifying anti-rheumatic drugs (DMARDs) of which methotrexate is most commonly used. ${ }^{2}$ In oligo-articular JIA, intra-articular steroid injections alone may be sufficient to control disease. In most other sub-groups, it has become standard practice to use a disease modifying agent (usually methotrexate) at diagnosis. Methotrexate takes 6 to 8 weeks to start to show an effect. Meanwhile, inflammation can be controlled with either intra-articular steroids or pulsed methylprednisolone depending on number and type of joints affected. The percentage of 'non-responders' to methotrexate can be as high as $30 \%$ or more depending on JIA-subtype. ${ }^{3,4}$ In 430 children with polyarticular 
Table I ILAR Classification of JIA'

\begin{tabular}{|c|c|}
\hline Category & Definition \\
\hline Systemic onset JIA & $\begin{array}{l}\text { a) Arthritis in I or more joints and } \\
\text { b) Fever of at least } 2 \text { weeks duration that is documented to be daily ('quotidian') and } \\
\text { c) One or more of } \\
\text { i. Evanescent erythematous rash } \\
\text { ii. Generalized lymph node enlargement } \\
\text { iii. Hepatomegaly and/or splenomegaly } \\
\text { iv. Serositis }\end{array}$ \\
\hline Oligoarticular JIA & $\begin{array}{l}\text { Arthritis affecting I to } 4 \text { joints during the first } 6 \text { months of disease } \\
\text { Two subgroups: } \\
\text { I. Persistent oligoarthritis: affecting } \leq 4 \text { joints throughout the disease course } \\
\text { 2. Extended oligoarthritis: affecting }>4 \text { joints after the first } 6 \text { months }\end{array}$ \\
\hline Polyarthritis (RF negative) & Arthritis affecting 5 or more joints during the first 6 months of disease; (RF negative) \\
\hline Polyarthritis (RF positive) & $\begin{array}{l}\text { Arthritis affecting } 5 \text { or more joints during the first } 6 \text { months of disease; } \\
2 \text { or more tests for RF are positive (at least } 3 \text { months apart) }\end{array}$ \\
\hline Psoriatic arthritis & $\begin{array}{l}\text { Arthritis and psoriasis, or arthritis and at least } 2 \text { of the following: } \\
\text { a) Dactylitis } \\
\text { b) Nail pitting and onycholysis } \\
\text { c) Psoriasis in a first-degree relative }\end{array}$ \\
\hline Enthesitis-related arthritis & $\begin{array}{l}\text { Arthritis and enthesitis, or arthritis or enthesitis with at least } 2 \text { of the following: } \\
\text { a) Sacroiliac joint tenderness and/or inflammatory lumbosacral pain } \\
\text { b) The presence of HLA-B27 antigen } \\
\text { c) Onset of arthritis in a male over } 6 \text { years of age } \\
\text { d) Acute (symptomatic) anterior uveitis } \\
\text { e) History of ankylosing spondylitis, enthesitis-related arthritis, sacroilitis with inflammatory } \\
\text { bowel disease, Reiter's syndrome or acute anterior uveitis in a first-degree relative. }\end{array}$ \\
\hline Undifferentiated arthritis & Arthritis that fulfils criteria in no category or in 2 or more of the above categories. \\
\hline
\end{tabular}

Abbreviation: RF, rheumatoid factor

JIA $72 \%$ responded as defined by ACR Pediatric 30 criteria but only $12 \%$ met the definition of complete disease control. ${ }^{3}$ Response rates are lower for children with systemic JIA and enthesitis-related arthritis. ${ }^{4}$ The advent of biologics has been of great importance in the management of children with refractory JIA who historically have suffered the greatest damage from unchecked inflammation and treatment side-effects.

Biologics describe a class of drug that has been designed to have biologic properties and include monoclonal antibodies, soluble cytokine receptors and recombinant receptor antagonists. ${ }^{2}$ This includes drugs that block TNF- $\alpha$ such as etanercept, infliximab and adalimumab as well as other agents such as anakinra, tocilizumab, abatacept and rituximab.

Etanercept is a recombinant fusion protein combining two human soluble p75 TNF receptors with an Fc domain of human IgG1 which binds TNF- $\alpha$ and $\alpha$-lymphotoxin reducing inflammation. It is given as a subcutaneous injection twice weekly at a dose of $0.4 \mathrm{mg} / \mathrm{kg}$ and is licensed and recommended by the Food and Drugs Administration (FDA) in USA in children over 2 years, the National Institute for Health and Clinical Excellence (NICE) in the UK, and the European Agency for the Evaluation of Medicinal Products (EMEA) of the EU in children over 4 years with polyarticular JIA unresponsive or intolerant to methotrexate. Until recently etanercept has been the only anti-TNF licensed in children and this together with its good long term efficacy and safety profile have made it the first choice anti-TNF treatment in most children with JIA that fail methotrexate. Adalimumab, another anti-TNF, has recently been licensed in several countries and offers an alternative. It is licensed in the UK for treatment of JIA in adolescents and in the USA for children 4 years and older. Other biologic therapies are used off license in certain circumstances.

The pathophysiology of JIA is complex. An imbalance between proinflammatory and anti-inflammatory elements of the immune system is described with TNF- $\alpha$ playing an 
important role in driving inflammation. This is confirmed in studies showing significantly elevated levels of serum and synovial fluid TNF- $\alpha$ and interleukin (IL)-2, IL-6, interferon (IFN) $\beta$ and IFN- $\gamma$ as well as high levels of synovial fluid IL$1 \alpha$ with corresponding impaired production of anti-inflammatory cytokines. ${ }^{5,6}$ Cytokine levels appear to correlate with disease activity including soluble TNF receptor levels. ${ }^{7}$

The morbidity of JIA can be marked and complications include pain, disability, deformity, growth failure, osteoporosis, macrophage activation syndrome, amyloidosis and even death. This affects the child's education, social functioning, psychological well-being and also has affects on the family.

Prior to publication of follow-up studies in JIA, there was a common viewpoint that JIA would often remit in adulthood. These studies challenge this, suggesting that JIA has a greater long-term consequence and progresses into adulthood more frequently than initially thought. ${ }^{8-12}$ Only patients under regular follow up in adulthood were included and thus these studies were biased towards the more severe end of the spectrum, excluding those patients with mild or resolved disease.

In one study, $42.9 \%$ of adults with JIA have severe functional limitations as measured by a Health Assessment Questionnaire (HAQ) score of $>1.5$. Those at greatest risk were patients with polyarticular disease course and in particular systemic JIA. 36.3\% were still taking a DMARD and $43.3 \%$ had clinically active disease. Adults with systemic onset JIA had accrued more damage from their disease. ${ }^{11}$ Adults with JIA achieved higher academic qualifications than their peers but they were more likely to be unemployed. . $^{810}$ $21.1 \%$ had suffered depression at some point and $31.9 \%$ had severe pain as measured on a visual analog scale. ${ }^{12}$

These follow-up studies provide further support to treat children with JIA 'early and aggressively'. A long-term follow-up study has demonstrated sustained benefits of early disease control. Even after 9 years, patients randomized to early sulfasalazine use versus placebo had a better outcome. ${ }^{13}$ This has to be balanced with the side-effects of treatments, many of which are relatively new and may have a long term safety profile that is not completely understood.

\section{Efficacy of etanercept in JIA}

It has become standard practice in therapeutic trials of JIA to measure efficacy using the American College of Rheumatology (ACR) Pediatric 30 (Pedi 30), Pedi 50, Pedi 70, Pedi 90, and Pedi 100 criteria for improvement based on the core set criteria. The core set of 6 responses include number of active joints, number of joints with limitation of movement, physician's assessment of disease activity (visual analog scale [VAS] 0-10 cm), parental assessment of patient's overall well-being (VAS), validated measure of physical function (Child Health Assessment Questionnaire [CHAQ]) and a laboratory measure of inflammation (Erythrocyte Sedimentation Rate, ESR). Definition of improvement for ACR Pedi 30 requires at least 30\% improvement from baseline in at least 3 out of 6 of the core set with no more than one of the core set worsening by $>30 \% .{ }^{14}$ ACR Pedi 50, 70, 90 and 100 are improvements from baseline of at least $50 \%, 70 \%, 90 \%$ or $100 \%$ respectively in at least 3 out of 6 core criteria and with no more than one of core set worsening by $>30 \%$. Outcome can also be assessed by radiological progression of disease and quality of life data.

The efficacy of etanercept in controlling disease activity in children with JIA has been demonstrated in one randomized control trial (RCT) and several non-randomized trials (Table 2). All studies except the initial open label extension and RCT used concomitant methotrexate.

In a two stage study, 69 patients aged 4 to 17 years with active polyarticular JIA despite treatment with methotrexate at $>10 \mathrm{mg} / \mathrm{m}^{2}$ entered an open label study where they all received subcutaneous etanercept at $0.4 \mathrm{mg} / \mathrm{kg}$ twice weekly. ${ }^{15}$ Sixty-four patients $(93 \%)$ completed treatment of 3 months. The response rate was 74\% (51/69) and was often rapid within the first few weeks of treatment. At the end of 3 months 64\% had an ACR50 response and 36\% ACR70 response.

The responders $(n=51)$ were eligible for a double-blind randomized control trial for 4 months. Significantly more patients flared in the placebo group compared to the etanercept group $(81 \%$ versus $28 \%, p=0.003)$ and the median time to flare was significantly greater in the etanercept versus the placebo group ( $>116$ days versus 28 days, $\mathrm{p}<0.001){ }^{15}$

At the end of the RCT $80 \%(20 / 25)$ of patients on etanercept achieved ACR30 compared with 35\% (9/26) in the placebo group. The median improvement in CHAQ was $54 \%$ compared with no obvious change in the placebo group. There was no significant difference in adverse events in the treatment group versus placebo. ${ }^{15}$

Efficacy and safety have been shown to continue in follow-up studies at 2, 4 and 8 years in 318 patient years of exposure. ${ }^{16-18}$ Children included in this study had severe refractory JIA with mean disease duration at recruitment of 5.9 years. This long disease duration may have resulted in a lower efficacy of etanercept than if it had been used earlier in the disease course. 
Table 2 Published studies on the use of etanercept in children with JIA;study populations described as polyarticular JIA include all those subtypes that had more than 4 joints involved and may include RF positive and RF negative JIA, psoriatic, systemic and enthesitis-related arthritis

\begin{tabular}{llll}
\hline Author(s), year & Number of patients & Study population & Study design \\
\hline Haapasaari et al $2002^{77}$ & 31 & Polyarticular JIA & Retrospective \\
Henrickson and Reiff $2004^{37}$ & 8 & ERA & Uncontrolled open label trial \\
Horneff et al $2008^{52}$ & 604 & All JIA & Prospective observational \\
Kietz et al $2002^{78}$ & 22 & Polyarticular JIA & Uncontrolled open \\
Kimura et al $2005^{29}$ & 82 & Systemic JIA & Retrospective questionnaire \\
Lahdenne et al $2003^{79}$ & 10 & Polyarticular JIA & Uncontrolled, open label \\
Lovell et al $2000^{15}$ & 69 & Polyarticular JIA & Open label trial \\
Lovell et al $2000^{15}$ & 51 & Polyarticular JIA & RCT \\
Lovell et al $2003^{16}$ & 43 & Polyarticular JIA & Uncontrolled extension trial \\
Lovell et al $2006^{18}$ & 34 & Polyarticular JIA & Uncontrolled extension trial \\
Lovell et al $2008^{17}$ & 26 & Polyarticular JIA & Uncontrolled extension trial \\
Prince et al $2008^{19}$ & 146 & All JIA except persistent oligoarticular & Prospective observational \\
Quartier et al $2003^{30}$ & 61 & Polyarticular JIA & Uncontrolled open label trial \\
Russo et al $2002^{31}$ & 15 & Systemic JIA & Prospective observational \\
Schmeling et al $200 I^{51}$ & 7 & All JIA except persistent oligoarticular & Case series \\
Takei et al $2001^{32}$ & 8 & Polyarticular JIA & Retrospective, high dose etanercept \\
\hline
\end{tabular}

Abbreviations: ERA, enthesitis-related arthritis; RCT, randomized controlled trial; RF, rheumatoid factor.

Although etanercept had good efficacy it did not lead to drug free remission in the majority of children. With time, most went on to receive methotrexate, alternative DMARDs or corticosteroids for disease flares. At baseline, no patients received DMARDS whilst during 8 years $53 \%$ received one or more DMARDs (38\% being methotrexate). Twenty-nine percent of patients received corticosteroids at the beginning of the open label extension which increased to $60 \%$ at some point during 8-year follow up. ${ }^{17}$

Sixty-six percent of patients withdrew during 8 years with the reasons stated as: parent or child refusal 14\%, suboptimal clinical response $12 \%$, physician decision $9 \%$, adverse events $7 \%$, lost to follow-up 5\%, protocol issues 5\% and other reasons $14 \%$. At 8 years response to treatment (for those still on treatment) was ACR30 83\% (40 of 48 patients), ACR50 77\% (36 of 47 patients), ACR70 61\% (28 of 46 patients), ACR90 41\% (19 of 46 patients) and ACR100 18\% (8 of 45 patients). ${ }^{17}$

Subsequent studies have demonstrated similar efficacy. An observational study of 146 patients with JIA (all subtypes) in a Dutch registry included 436 patient years of exposure. At 3 months of treatment, an ACR 30 was seen in $77 \%$ of patients. The response was sustained and $36 \%$ of all patients met remission criteria. $67 \%$ of patients at 15 months had discontinued corticosteroid treatment. One third of patients remained on etanercept as a single agent. ${ }^{19}$

A small study of 21 children with polyarticular and systemic JIA showed that starting etanercept had a significant impact on functional status, emotional well being and quality of life scores. ${ }^{20}$

In addition to improving clinical outcomes, a study of 40 patients with polyarticular JIA included in an Italian registry has suggested that etanercept improves radiological progression of disease as measured by Poznanski score (carpo-metacarpal ratio) at baseline and 1 year. ${ }^{21}$

Etanercept has been shown to improve growth velocity in children with JIA. The most significant predictor of growth rate was the degree of inflammation, even after the reduction of corticosteroid dose was taken into account suggesting that improved growth on etanercept is secondary to reduced inflammation. ${ }^{22}$ This was confirmed in another study that showed significant increase in height velocity after etanercept was commenced in prepubertal children. In pubertal children, height increase on etanercept did not reach statistical significance, but this may have been due to an underpowered study. ${ }^{23}$

\section{Challenges of using etanercept in children with JIA Do all subsets of JIA respond equally
well to etanercept?}

There are a growing number of data available on safety and efficacy of etanercept for use in polyarticular JIA, but results may not be generalizable to all disease sub-types. Systemic onset JIA (sJIA), although less common than 
other sub-groups can be very challenging to treat. Trials that include this sub-group tend to select patients that have a polyarticular course, but no significant systemic symptoms at time of treatment. There is a paucity of evidence for the use of etanercept in other sub-groups such as psoriatic JIA and enthesitis-related arthritis.

\section{Etanercept in systemic JIA}

Increasing evidence from trial and registry data suggests that children with sJIA do not respond as well to etanercept as children with polyarticular disease. There are growing data that SJIA is driven by other pro-inflammatory cytokines other than TNF, such as IL-1, IL-6 and IL-18. ${ }^{24-26}$ Early reports of the use of anakinra (1L-1RA) ${ }^{26}$ and tocilizumab (anti-IL-6 monoclonal antibody ${ }^{27}$ show promise in this sub-group of JIA.

The RCT of etanercept in JIA showed that patients with sJIA had a higher rate of disease flare (44\%) than other subsets. ${ }^{15}$ A long-term follow up study of the Dutch national register demonstrated that patients with sJIA responded less well than other subtypes across ACR30, 50 and 70 responses $(\mathrm{p}<0.01)$ and withdrawal secondary to poor clinical response was also more likely $(\mathrm{p}<0.001) .{ }^{19}$ Although response to etanercept was less predictable in children with sJIA, those that did respond showed similar efficacy to other groups in the long-term, with $38 \%$ of all systemic patients entering remission on etanercept. ${ }^{19}$ Since this was an observational study, patients may have been on other concomitant medications that may have made a difference to their outcome.

Other authors have documented similar disappointing response rates in this sub-group. An analysis of 322 patients with JIA treated with etanercept and monitored within the German registry has demonstrated lower therapeutic efficacy of etanercept in children with sJIA compared to other subgroups. ${ }^{28}$

A retrospective study of 82 patients with active and refractory sJIA showed a significant decrease in active joint count, ESR, physician global VAS and the number of patients on prednisolone ( $46 \%$ ceasing need for corticosteroids). However, $54 \%$ had a poor response to etanercept and $45 \%$ had at least one flare. $35 \%$ of patients discontinued etanercept, mostly due to poor response or disease flare. ${ }^{29}$ This study is limited by the retrospective standardized questionnaire methodology and poor response rate. An open label, prospective, multi-center study by Quartier et al also demonstrated a higher rate of treatment failure in sJIA compared to other subgroups. ${ }^{30}$

In another small observational study of 15 patients with refractory sJIA treated with etanercept in combination with methotrexate, an initial response was seen, but was only sustained in $20 \%$ of patients. In others, increasing flares and progressive loss of effectiveness was seen over time. ${ }^{31}$ The use of high dose etanercept $(0.8 \mathrm{mg} / \mathrm{kg} / \mathrm{dose})$ had a beneficial effect in 2 out of 5 patients with sJIA failing to respond to conventional doses. ${ }^{32}$ Thus, dose modifications may be required in patients with active sJIA.

Etanercept is tolerated in this sub-group of JIA with few adverse effects. There has been one report of commencement of etanercept and the development of macrophage activation syndrome in a patient with sJIA. ${ }^{33}$ Patients with sJIA are at risk of developing this complication and it is unlikely that etanercept was causal in this case but may represent the inability of etanercept to control disease. Also, etanercept has been used successfully in the treatment of macrophage activation syndrome. ${ }^{34}$

In order to determine accurate efficacy of etanercept in sJIA, prospective multi-center RCTs are needed using validated response criteria for sJIA that could incorporate other features of disease as well as joint count and core set, such as fever, rash, anemia, pericarditis, or macrophage activation syndrome. Current JIA research or registry data do not often capture systemic features such as rash or fever.

\section{Etanercept and enthesitis-related arthritis (ERA)}

Adult data of patients with ankylosing spondylitis have shown etanercept to be efficacious and well tolerated in several RCTs. ${ }^{35,36}$

Pediatric data are limited to uncontrolled studies, but suggest that etanercept may be efficacious for children with ERA. In a pilot study of 8 patients with active ERA, all had improvement in active joint count. Four out of the 8 patients had enthesitis which completely resolved on treatment. This clinical improvement was sustained over a 2-year follow-up period. ${ }^{37} \mathrm{~A}$ further case report showed rapid and sustained clinical improvement, with radiological remission at 2 years demonstrated by magnetic resonance imaging. ${ }^{38}$

\section{Extended oligoarticular JIA}

Extended oligoarthritis can be associated with a worse prognosis compared to other sub-groups of disease and early aggressive treatment is warranted..$^{39}$ An observational study of 61 patients looking at efficacy of etanercept in different sub-groups of JIA indicated similar response rates in extended oligoarticular and polyarticular subgroups. ${ }^{30}$ Other studies have included children with extended oligoarticular JIA and have shown good efficacy to etanercept. ${ }^{19}$ 


\section{Etanercept and psoriatic arthritis}

Etanercept has been shown to be efficacious in adult patients with psoriatic arthritis with improvement of skin and joint symptoms and slowed radiographic progression..$^{40}$ Trials in children with psoriatic JIA are lacking. Data from the German biologics registry of 11 children with psoriatic JIA showed the response rate to etanercept was comparable with polyarticular JIA with more than $80 \%$ reaching ACR Pedi 30 response versus ACR Pedi 30 response of in those with polyarticular JIA. ${ }^{41}$ These rates were also observed in the Dutch registry in 8 patients. ${ }^{19}$

\section{Uveitis}

The risk of developing uveitis in children with JIA is approximately $10 \%$ to $25 \%$. Twenty-four percent of JIA patients who develop uveitis will develop ocular complications, of which cataracts is the most common. ${ }^{42}$ Topical corticosteroids remain first line treatment, followed by addition of DMARDs such as methotrexate, ciclosporin A or mycophenolate mofetil, progressing to anti-TNF therapy for resistant disease.

Trials of etanercept versus placebo show conflicting results. An underpowered double blind RCT of 12 patients did not detect any difference in ocular inflammation between placebo and etanercept $\mathrm{t}^{43}$ and a retrospective study showed no significant change in number of flares of uveitis before or after etanercept therapy. ${ }^{44}$ However, a small prospective study showed a statistically significant improvement in 10/16 eyes $(63 \%)$ with improvements in visual acuity in $40 \%$. Those who did not initially respond showed no improvement with an increased dose of etanercept. ${ }^{45}$

In a small non-randomized trial of patients with refractory JIA started on anti-TNFs, patients on infliximab had greater reduction in inflammatory activity of uveitis compared to those treated with etanercept $(\mathrm{p}=0.047)$ and experienced less flares of uveitis $(p=0.015) .5 / 24$ patients on etanercept developed new onset uveitis. ${ }^{46}$ In patients with refractory uveitis, $32 \%$ had no response to etanercept as measured by $\leq 50 \%$ reduction in oral and topical corticosteroids, whereas all patients in the infliximab group showed $\geq 50 \%$ reduction in oral or topical corticosteroids, reaching statistical significance. $35 \%$ of patients on etanercept were changed on to infliximab and a response was noted. Three patients who did not respond to infliximab had a good response to adalimumab. The mean time of duration of uveitis before starting anti-TNF treatment was 52 months. ${ }^{47}$

Children who show a poor response to one agent may benefit from change to another anti-TNF. Currently, treatment of JIA associated uveitis with anti-TNF therapy is reserved for those with resistant disease and response may be greater in a population with lower duration of inflammation and less damage from uveitis and treatment. The efficacy of etanercept in patients with JIA associated uveitis is yet to be determined and requires robust randomized, placebo control trials or head to head trials with other anti-TNFs. Meanwhile, small studies suggest that infliximab and potentially adalimumab have better efficacy in this group.

\section{Under 4 years of age}

Etanercept is licensed for use in children with JIA who are over the age of 4 in Europe and the UK, while in the US it is licensed in children over 2 years of age. There is only one observational study that looks at etanercept efficacy and safety in children below the age of 4 years. Twenty-five children with JIA were identified from the German registry. There were no serious adverse events (SAE) identified. The authors compared data with those from older children and found no major differences in safety or efficacy despite a higher proportion of children less than 4 years of age having sJIA, higher disease activity and more frequent corticosteroid and methotrexate use at baseline. ${ }^{17,48}$ The authors conclude that the cut off of 4 years is artificial with no clear medical basis.

\section{What to do when etanercept fails?}

The effect of etanercept is often seen within 2 weeks, although full response takes longer to achieve. If a significant effect is not seen within 3 to 4 months, then the patient is considered to be a non-responder to the treatment at that dose. When etanercept does not adequately control disease there are several options available including using combination therapy (etanercept and methotrexate), optimizing conventional treatments (changing methotrexate to sub-cutaneous administration or increasing dose), maximizing etanercept dose or changing to an alternative biologic therapy.

\section{Combination therapy}

Studies in adult patients with rheumatoid arthritis (RA) have shown an increased response rate in those treated with combination therapy of etanercept and methotrexate compared to etanercept alone. ${ }^{49,50}$ A pilot study in children with refractory JIA showed combination therapy appeared to be tolerable and may enhance efficacy. ${ }^{51}$ Another prospective study in children with JIA included 504 patients on combination therapy and 100 patients on etanercept alone. There was no significant difference in discontinuation rates in the two groups, suggesting similar tolerability. There was significant 
difference in response rates at 1 year with an ACR30 of $70 \%$ in etanercept and $81 \%$ in combination group $(p<0.05)$ and an ACR70 of $45 \%$ versus $62 \%$ respectively $(p<0.01)$. There was no significant difference in the number of $\mathrm{AE}$ reported between the two groups. Infectious SAE were more frequent in the combination group but this did not reach statistical significance. On subgroup analysis, patients with RF negative polyarticular JIA had a significantly higher response to combination therapy versus monotherapy but no statistically significant difference was observed in systemic or psoriatic arthritis subgroups. There were important differences in the two groups at baseline with the combination group having a significantly higher proportion of RF positive polyarticular disease, less enthesitis-related disease and more corticosteroid use than the single agent group. ${ }^{52}$

\section{High dose etanercept}

In patients who have had an inadequate response to etanercept at $0.4 \mathrm{mg} / \mathrm{kg}$, the dose can be doubled to $0.8 \mathrm{mg} / \mathrm{kg} / \mathrm{dose}$ (to a maximum of $25 \mathrm{mg}$ ). Small studies evaluating this have not documented a definite improvement. In a study of 8 patients with active polyarticular disease unresponsive to at least 3 months of standard dose treatment, etanercept was increased to at least $0.8 \mathrm{mg} / \mathrm{kg} /$ dose. Only 2 out of 8 patients showed a clinical response but 5 out of 8 patients had systemic JIA which may not respond as well to etanercept as other JIA subtypes. ${ }^{32}$ In another study, 7 out of 15 (47\%) patients with sJIA discontinued etanercept due to lack of response even after increasing dose to $1 \mathrm{mg} / \mathrm{kg} / \mathrm{dose} .{ }^{31}$

There does not appear to be any increase in AE at high dose. ${ }^{29}$ High dose etanercept appears to be well tolerated but may not lead to an increase in efficacy.

\section{Once-weekly etanercept}

A prospective pilot study of 12 patients showed no change in efficacy in those changed to once weekly administration of etanercept at $0.8 \mathrm{mg} / \mathrm{kg} / \mathrm{dose}$. $83 \%$ responded to etanercept with an ACR30 score and 50\% achieved ACR70. Eighty-three percent were on concomitant methotrexate. No SAE or serious infections were noted. ${ }^{53} \mathrm{~A}$ study of 11 patients in remission changed to once-weekly dose of $0.8 \mathrm{mg} / \mathrm{kg}$ and 2 patients initiated on $0.8 \mathrm{mg} / \mathrm{kg}$ once weekly showed that this dosing regime retained and induced remission in the short term. ${ }^{54}$

In children who find it difficult to adhere to twice weekly injections this pilot suggests a similar efficacy and safety profile to twice weekly administration. Pharmacokinetic models in children with JIA showed similar profiles for once weekly versus twice weekly etanercept. ${ }^{55}$

\section{Safety of etanercept in JIA}

In the initial RCT of etanercept there was no significant difference between the rates of adverse events in the etanercept versus the placebo group. The most frequent adverse events were injection site reactions (39\%), upper respiratory tract infections (35\%), headaches (20\%), abdominal pain (16\%), vomiting (14\%), nausea (12\%) and rash (10\%). ${ }^{15}$

A recent 8-year open label extension trial of etanercept collected 318 patient years of exposure. $23 \%$ of the original RCT reported SAE representing 0.12 SAE per patient year. Reassuringly, there was no increase in the rate of SAE with time. Only $7 \%$ of patients discontinued etanercept because of adverse events. No cases of systemic lupus erythematosis (SLE), demyelination, tuberculosis (TB), opportunistic infection, malignancy or death were identified. ${ }^{17}$

In a longitudinal study, SAE were 0.029 per patient year with no difference in SAE between subgroups of JIA. $4 \%$ of patients discontinued etanercept because of SAE. ${ }^{19}$ Another study showed no difference in rate of SAE between subgroups but $20 \%$ discontinued etanercept due to $\mathrm{SAE}^{30}$

\section{Injection site reactions}

Injection site reactions are common (28\%-39\%) but do not usually cause cessation of the drug. ${ }^{15,30}$ They may be an example of a T-lymphocyte mediated delayed-type hypersensitivity reaction and become less frequent over time due to eventual induction of tolerance. ${ }^{56}$ They do not tend to need any intervention but if symptomatic, they can be helped by application of ice or cold compresses, or use of topical corticosteroid or antihistamine to the injection site post injection.

\section{Infections}

Anti-TNF therapies have been associated with serious manifestations of common bacterial infections (such as skin abscesses and cellulitis) as well as atypical or opportunistic infections such as TB, coccidiomycosis, listeriosis, histoplasmosis and aspergillosis. ${ }^{57}$ In pediatric patients with JIA treated with etanercept the overall infection rate was $22.8 \%$, of which 32 were minor infections and 2 serious infections (prosthetic hip infection and bacterial pneumonia). ${ }^{58}$ Lovell et al reported 9 cases of medically important infections (MMIs) in 8 patients over 8 years (exposure adjusted rate 0.03 events per patient year). These included pyelonephritis, peritonitis and appendicitis, aseptic meningitis secondary to varicella, acute varicella infection, soft tissue infection, postoperative wound infection, sepsis requiring foot amputation, dental abscess and gastrointestinal infection. There was no increase in the rate of MMIs with time. ${ }^{17}$ 
Data from the UK biologics registry in adults with RA showed that patients on etanercept had a lower rate of serious lower respiratory tract infections than infliximab and adalimumab-treated patients. ${ }^{59}$

Reactivation of TB is a serious risk in patients commenced on anti-TNFs; therefore local guidelines for TB screening should be followed. Patients on anti-TNFs have impaired granuloma formation allowing mycobacterium spread. In such patients TB infection is frequently extrapulmonary or disseminated. The risk of TB with anti-TNFs appears to be higher in those treated with infliximab than etanercept and this in part may be explained by the difference in apoptotic properties between the two drugs. Adults patients with RA developed TB earlier on infliximab (median time 3 months) than on etanercept (median time 11.5 months). ${ }^{57}$ However, only one case of TB has been reported in children with JIA and that child received infliximab after etanercept therapy. ${ }^{19}$

Varicella infection can be more severe in children on DMARDs. In the studies of children on etanercept for JIA, 4 children developed varicella in 258 patient treatment-years. Of these, 3 stopped etanercept temporarily and one continued. There were no complications. ${ }^{58}$ During an extension study 3 children were hospitalized due to varicella. ${ }^{16}$

Prior to starting anti-TNF treatment children should be screened for TB as per local/national guidelines and have their varicella and measles immunity checked. In children who are non-immune, vaccination is recommended if not contraindicated by other DMARD use and if clinical status allows delay in starting anti-TNF treatment. In non-immune children who can not be vaccinated, parents should be advised about what action to take if their child comes into contact with varicella.

\section{Autoimmune disease and autoantibodies}

Etanercept use in JIA has been linked with the development of autoantibodies and rarely with clinical manifestations of systemic lupus erythematosis (SLE) ${ }^{60,61}$ There have also been reports of the development of sarcoid, inflammatory bowel disease, demyelination and uveitis while on etanercept. ${ }^{19,58,62}$

The difficulties with interpretation of these studies is identifying whether there is a true causal link between anti-TNFs and autoimmune disease rather than association only. In published studies, baseline autoantibody status may not be stated and it may not be clear whether patients had symptoms of autoimmune disease prior to commencing antiTNFs. However, if symptoms resolve on ceasing the drug and return on re-challenge this provides a strong suggestion of causality.
Clinicians should remain vigilant for clinical evidence of emerging new autoimmune disease and check laboratory titres of autoantibodies if concerned. In the event of clinically significant disease, etanercept may need to be discontinued.

A prospective study of 26 patients with JIA on anti-TNF agents for at least 2 years found that $23 \%$ developed persistently raised autoantibodies. Of those patients on etanercept $(n=14)$, half had positive anti-nuclear antibody (ANA) at baseline and 5 developed new autoantibodies. Four patients developed anti-R1 antibodies and 1 patient developed anti-thyroid antibodies associated with Hashimoto's thyroiditis (etanercept was withdrawn and treatment with thyroxine was ceased 6 months afterwards). There was no other link with development of new autoantibodies or change in autoantibody titers with clinical disease or flares of arthritis. ${ }^{61}$ This is confirmed by Lovell et al where autoantibody status in 43 children showed that no child who was ANA negative initially became consistently positive, and none developed anti-double stranded (ds-DNA) or antiphospholipid antibodies. ${ }^{16}$ However 2 patients (1.6\%) on etanercept developed anti-dsDNA without clinical sequalae in a subsequent study. ${ }^{58}$

There have been two reports of the development of SLE in children with JIA treated with etanercept. A child who was previously ANA positive 1:160 and dsDNA negative commenced etanercept therapy with a rise in ANA titers to 1:2560. He developed temperature, urticaria and swollen hands with anti-dsDNA becoming 1:320. Etanercept was withdrawn but symptoms did not resolve until corticosteroids were commenced. These were weaned within a couple of months. ${ }^{63}$ Another patient developed SLE while on etanercept manifest as positive anti-dsDNA antibodies, fatigue, hair loss, type IV glomerulonephritis and severe lung disease. Her disease was not reversible on withdrawing etanercept. ${ }^{60}$

A review of autoimmune disease induced by anti-TNFs identified 37 reported cases of lupus in adults treated with etanercept. Many had clinical features of drug induced lupus rather than systemic lupus erythematosis with positive autoantibodies and constitutional, cutaneous and musculoskeletal symptoms only. Etanercept was withdrawn in $94 \%$ and 36/37 showed improvement. However, some required treatment with steroids and/or other DMARDs. Of note $80 \%$ of patients had RA and some had pre-existing features of lupus, suggesting an overlap syndrome. The authors conclude that etanercept may cause drug-induced lupus or trigger underlying SLE. ${ }^{64}$

The same review identified 59 cases of vasculitis in adult patients on etanercept, $89 \%$ were cutaneous but $29 \%$ had organ involvement. ${ }^{64}$ 
Two out of 146 patients developed sarcoid on etanercept ${ }^{19}$ and a single case of new onset diabetes mellitus type 1 after 5 months of etanercept therapy has been reported. ${ }^{65}$

Overall, the incidence of auto-antibody formation seems to occur less frequently in children compared to adults ${ }^{61}$ and therefore it may follow that the development of autoimmune disease may also be less.

\section{Neuropsychiatric}

Neuropsychiatric side effects were not recognized in the initial trial but with adverse event reporting via national registries these have been increasingly identified. An Italian study reported the frequency of neuropsychiatric adverse events at $28.1 \%$ and included headaches, anxiety, depression, aggression, pain amplification syndromes, fatigue and vertigo. Severe headache, aggression and pain amplification syndromes appeared to be dose dependent, occurring only when etanercept dose was increased above $0.4 \mathrm{mg} / \mathrm{kg}$ and remitting on return to lower dose. Also reported was a case of hypoglossal paralysis without suggestion of demyelination or vasculitis on MRI imaging. All neuropsychiatric manifestations resolved on stopping or reducing etanercept although some required treatment with drugs. ${ }^{58}$ The frequency of severe psychiatric disorder in another study was $2 / 61 .^{30}$ However, these data come from open label trials and therefore can not provide information on the incidence of neuropsychiatric symptoms in etanercept compared to a control group.

\section{Etanercept and inflammatory bowel disease}

Infliximab has been shown to be effective in the treatment of Crohn's disease whilst etanercept has not. ${ }^{66}$ Infliximab is a chimeric human murine monoclonal antibody to TNF- $\alpha$. Both etanercept and infliximab bind soluble TNF- $\alpha$ but only infliximab binds to membrane bound TNF. Binding of infliximab to activated lamina propria $T$ cells induces apoptosis. These differences may help to explain why infliximab is a successful treatment for inflammatory bowel disease (IBD) while etanercept is not. In fact, etanercept has been associated with the development of new-onset IBD.

Of JIA patients on etanercept, 3.9\% $(\mathrm{n}=5)$ developed new onset inflammatory bowel disease. ${ }^{58}$ Three had biopsies which confirmed IBD and the other two had symptoms that resolved rapidly on cessation of etanercept. All had long standing JIA with no previous bowel symptoms to suggest arthritis secondary to IBD although IBD-related arthritis may present prior to bowel symptoms. All bowel symptoms responded to a change of treatment to infliximab. ${ }^{58}$ This is confirmed in other data sets where 2 patients out of $146(1 \%)$ developed $\mathrm{IBD}^{19}$ and 1/61 developed bloody diarrhea and abdominal pain on etanercept. ${ }^{30}$ In 2 cases symptoms stopped on withdrawal of treatment and returned with reintroduction of etanercept. Both patients had histological confirmation of Crohn's disease. ${ }^{30,67}$ These findings need to be confirmed in larger trials but show the importance of registries in detecting rarer adverse events associated with etanercept which were not discovered in original studies.

\section{Demyelination}

On review of the FDA Adverse Events Reporting System, 17 patients who received etanercept for inflammatory arthropathies developed features in keeping with demyelination with the majority having associated MRI findings. Symptoms included, most commonly, paresthesia, visual disturbance due to optic neuritis, confusion, gait disturbance, facial palsy and Guillain-Barré syndrome. All events were temporally related to etanercept and all improved or resolved on withdrawal of etanercept. One patient had a relapse of neurological symptoms on restarting etanercept. Two patients had JIA but were age 21 at onset of symptoms ( 1 had paresthesia with cervical myelitis on MRI, the other had optic neuritis confirmed on MRI) ${ }^{62}$

On open label extension of the initial Lovell study, with 318 patient years of exposure, there were no cases of demyelination. ${ }^{17}$ Likewise, no cases were identified within the Dutch registry with 436 years of exposure. ${ }^{19}$ The German registry had 1 suspected case of demyelination out of 610 patients. ${ }^{52}$

\section{Tumors}

There have been a few case reports of malignancy in children treated with etanercept. So far, long term data suggest that there is no increase in incidence in tumors over time, but the question of malignancy risk in children on etanercept remains unanswered and needs close ongoing observation. It is very difficult to study the malignancy risk in this group as there are no appropriate reference groups for comparison to children on etanercept and effects of anti-TNFs may only become apparent in longer term studies. Importantly, US studies of 318 patient years of exposure and Dutch registry data with 436 patient years of exposure both identified no cases of malignancy. ${ }^{17,19}$ Data from the German JIA registry identified 3 malignant tumors (yolk sac tumor after 3 weeks of treatment, thyroid carcinoma in a 17 year old treated with etanercept for 10 months and non-Hodgkin's lymphoma in a patient also 
on methotrexate, azathioprine and ciclosporin A). ${ }^{52}$ In data including 258 patient treatment-years, there was one case report of a 25-year-old female with long-standing RF positive polyarticular disease who had also received multiple other DMARDS (including methotrexate and infliximab) developing thyroid cancer whilst on etanercept. ${ }^{58}$

Worldwide safety data collected on all patients who have received anti-TNFs identified 39 cases of leukemia associated with etanercept but the majority of these patients were adults with RA, where RA is known to be a risk factor for hematological malignancy. ${ }^{68}$ Adult data of patients with RA on anti-TNFs in 89,710 person-years of observation found no increased risk of lymphoma compared to patients not treated with anti-TNF therapy. ${ }^{69}$

\section{Other adverse events}

A case report of a patient with polyarticular JIA on methotrexate and etanercept is described who developed thymic enlargement, cervical and hilar lymphadenopathy with left sided pleural effusion. Biopsy excluded malignancy and confirmed true thymic hyperplasia. Infection was also excluded. Clinical features resolved on stopping etanercept and methotrexate although methotrexate was later restarted. ${ }^{70}$

\section{Death}

On literature review only 3 reports of deaths in patients with JIA on etanercept could be identified. All were patients with systemic disease who received multiple immunosuppressive agents and had uncontrolled disease despite etanercept. Etanercept had not been used for at least 8 months. One patient also received infliximab and died from TB, one had suspected sepsis and one died from macrophage activation syndrome. ${ }^{19}$

\section{Challenges for the future}

Despite increasing data on the use of etanercept in JIA and adult RA there remain several unanswered questions.

\section{Which biologic therapy should be used in which JIA sub-group?}

Currently, etanercept tends to be the first choice biologic for treatment of resistant disease in most sub-groups of JIA. Although good efficacy is demonstrated in polyarticular JIA, there are currently insufficient data on other sub-groups and head to head trials of different biologics are lacking. Preliminary studies suggest that etanercept is less efficacious than other biologics in systemic onset JIA and in uveitis. It also has limited efficacy in Crohn's disease, sarcoid, and Wegener's granulomatosis. Differences can perhaps be explained by different drug characteristics, binding properties, pharmacokinetics, dosing patterns, and possibly by pathophysiology of disease type. Etanercept (but not infliximab) is known to bind lymphotoxin which is important in JIA, immune defence and immune development. On the other hand, bolus administration of infliximab leads to a greater variability of drug concentration over time, but better tissue penetration at higher peaks. ${ }^{71}$ This increased penetration may explain why infliximab seems to be better than etanercept in the control of uveitis, Crohn's disease and vasculitis, although further studies are needed.

At present, we are unable to predict accurately which child will respond to any particular biologic. Perhaps this will change in the future with a better understanding of genetic and cytokine profiles for different sub-groups. For example, work on sJIA has suggested that although TNF- $\alpha$ levels are raised, they are out of phase with the fever. In contrast, IL- $1 \alpha$ and IL-6 may be more important in the pathogenesis of sJIA. ${ }^{72}$ Early studies suggest a good response to IL-1 $\alpha$ blockade with anakinra ${ }^{26}$ and IL-6 blockade with tocilizumab. ${ }^{27}$ In contrast, patients with polyarticular JIA respond less well to anakinra. ${ }^{73}$

\section{Is there benefit in changing biologic if anti-TNF therapy fails?}

There is evidence to suggest that switching from one antiTNF to another is a reasonable therapeutic option in children with JIA. ${ }^{74}$ In addition, therapeutic trials of newer biologics, such as abatacept, are including children with JIA who have failed anti-TNF therapies. ${ }^{75}$ This raises questions regarding safety of treating children with consecutive biologics and drug wash-out times, particularly with use of biologic agents such as rituximab that can cause B cell depletion for months or even years.

It is not known whether the use of multiple biologics over time leads to a cumulative risk of immune modulation, malignancy or other events in later life. This is particularly important in children who are exposed to biologic therapies in their early years and may be at risk of yet unknown consequences. However, these potential risks need to be balanced against the known risk of disability and damage from uncontrolled disease.

Use of multiple biologics has long-term funding implications. Economic analysis needs to balance use of biologic therapies with the cost of long-term disability and morbidity including funding for joint replacement surgery, inability to work, loss of income and payment of disability benefits. 


\section{How long should children with JIA remain on biologic therapy?}

At present there are few data on withdrawal of drug therapy in JIA. Most clinicians continue to treat a child until they have had at least 1 year symptom-free period before trying to stop a DMARD or biologic. It is unclear whether it is beneficial to reduce dose or frequency of drug administration gradually rather than simply stopping biologic therapy. Data from the Dutch registry of 17 patients who discontinued etanercept due to good response showed that 53\% $(n=9)$ did not flare (mean follow up 1.4 years) and that these patients were in remission longer before stopping therapy than those that did flare $(1.9$ versus 0.3 years; $p<0.01)$. All children that ceased etanercept immediately $(n=3)$ flared. The rest tapered their dose before stopping. ${ }^{76}$ Studies are needed to evaluate further when and how to stop biologics and what proportion of children will flare on cessation.

\section{Should etanercept be used for refractory disease or at start of treatment?}

Currently, use of etanercept and other biologics is reserved for refractory JIA unresponsive to methotrexate or for children who fail to tolerate methotrexate. It can be hypothesized that disease remission may be achieved earlier by aggressive use of a combination of steroid, methotrexate and etanercept at diagnosis of JIA, but there is no evidence to confirm this. In order to answer this question, recruitment is currently taking place in the US for a randomized double blind placebo controlled trial of early aggressive therapy in polyarticular JIA (TREAT in JIA) comparing weekly subcutaneous methotrexate, oral prednisolone and once weekly etanercept $0.8 \mathrm{mg} / \mathrm{kg}$ with methotrexate, oral prednisolone and placebo etanercept at onset of disease (http://clinicaltrials.gov/ct2/show/record/NCT00443430).

\section{Are biologic therapies safe in children?}

Initial studies in adults and children suggest that etanercept and other biologics are efficacious and safe in the treatment of arthritis. However, with increasing use, potential risks and associations are being identified that were not apparent in initial trials. Registries are extremely important to determine safety of drug use over time, particularly in children. One current difficulty that needs to be overcome is how to deal with longterm monitoring when children transfer to adult care since up to now separate registries have been kept for JIA and RA.

\section{Conclusions}

The available evidence suggests that etanercept is a safe and efficacious drug for the treatment of JIA. It is generally well tolerated with a small proportion of serious adverse events and medically important infections. When using etanercept or other biologics, it is important to consider other factors that may contribute to adverse events, infections and malignancy such as longstanding inflammatory disease, co-morbidities and the frequent use of concomitant DMARDs. Current safety data are taken from randomized controlled trials and longitudinal observational studies that include a cohort of patients with longstanding refractory disease that may be more prone to adverse events from the nature of their disease. In recent years, there has been a tendency to treat patients more aggressively earlier in their disease course. It may be by doing this that patients are at less risk of adverse events and are able to achieve disease remission within a shorter time scale. On the other hand, resistant cases may be exposed to cumulative risks from the use of multiple biologic therapies over time. The long term safety profile remains unclear, particularly in relation to serious adverse events such as malignancy, infection and demyelination in a pediatric age group who may have long exposure to anti-TNF therapy from a very early age. Continued careful documentation of adverse events within registries is crucial, with a remit to follow children into late adulthood.

Published studies looking at the impact of juvenile idiopathic arthritis in adult life include patients who were treated as children before the advent of biologics. Hopefully, the outlook for future populations of JIA patients will be better.

Despite an increasing evidence base for the use of etanercept and other biologics in JIA, there are still unanswered questions. Further trials are needed and head to head trials comparing biologics in different sub-groups of disease would be beneficial. National and international collaboration is necessary to achieve sufficient patient numbers to make trials meaningful, particularly for sub-group analysis. Over time, a greater insight into the genetic and cytokine variability of different JIA sub-groups may lead to a better understanding of treatment response and enable more targeted treatment for individuals. Meanwhile, etanercept has become an established and dependable treatment for methotrexate-resistant JIA.

\section{Disclosures}

The authors declare no conflicts of interest.

\section{References}

1. Petty RE, Southwood TR, Manners P, et al. International League of Associations for Rheumatology classification of juvenile idiopathic arthritis: second revision, Edmonton, 2001. J Rheumatol. 2004;31(2):390-392.

2. McCann LJ, Woo P. Biologic therapies in juvenile idiopathic arthritis why and for whom? Acta Reumatol Port. 2007;32(1):15-26. 
3. Ruperto N, Murray KJ, Gerloni V, et al. A randomized trial of parenteral methotrexate comparing an intermediate dose with a higher dose in children with juvenile idiopathic arthritis who failed to respond to standard doses of methotrexate. Arthritis Rheum. 2004;50(7):2191-2201.

4. Woo P, Southwood TR, Prieur AM, et al. Randomized, placebocontrolled, crossover trial of low-dose oral methotrexate in children with extended oligoarticular or systemic arthritis. Arthritis Rheum. 2000;43(8):1849-1857.

5. Kutukculer N, Caglayan S, Aydogdu F. Study of pro-inflammatory (TNF-alpha, IL-1alpha, IL-6) and T-cell-derived (IL-2, IL-4) cytokines in plasma and synovial fluid of patients with juvenile chronic arthritis: correlations with clinical and laboratory parameters. Clin Rheumatol. 1998;17(4):288-292.

6. Lepore L, Pennesi M, Saletta S, Perticarari S, Presani G, Prodan M. Study of IL-2, IL-6, TNF alpha, IFN gamma and beta in the serum and synovial fluid of patients with juvenile chronic arthritis. Clin Exp Rheumatol. 1994;12(5):561-565.

7. Mangge H, Kenzian H, Gallistl S, et al. Serum cytokines in juvenile rheumatoid arthritis. Correlation with conventional inflammation parameters and clinical subtypes. Arthritis Rheum. 1995;38(2):211-220.

8. Foster HE, Marshall N, Myers A, Dunkley P, Griffiths ID. Outcome in adults with juvenile idiopathic arthritis: a quality of life study. Arthritis Rheum. 2003;48(3):767-775.

9. Packham JC, Hall MA. Long-term follow-up of 246 adults with juvenile idiopathic arthritis: social function, relationships and sexual activity. Rheumatology (Oxford). 2002;41(12):1440-1443.

10. Packham JC, Hall MA. Long-term follow-up of 246 adults with juvenile idiopathic arthritis: education and employment. Rheumatology (Oxford). 2002;41(12):1436-1439.

11. Packham JC, Hall MA. Long-term follow-up of 246 adults with juvenile idiopathic arthritis: functional outcome. Rheumatology (Oxford). 2002;41(12):1428-1435.

12. Packham JC, Hall MA, Pimm TJ. Long-term follow-up of 246 adults with juvenile idiopathic arthritis: predictive factors for mood and pain. Rheumatology (Oxford). 2002;41(12):1444-1449.

13. van Rossum MA, van Soesbergen RM, Boers M, et al. Long-term outcome of juvenile idiopathic arthritis following a placebo-controlled trial: sustained benefits of early sulfasalazine treatment. Ann Rheum Dis. 2007;66(11):1518-1524.

14. Giannini EH, Ruperto N, Ravelli A, Lovell DJ, Felson DT, Martini A. Preliminary definition of improvement in juvenile arthritis. Arthritis Rheum. 1997;40(7):1202-1209.

15. Lovell DJ, Giannini EH, Reiff A, et al. Etanercept in children with polyarticular juvenile rheumatoid arthritis. Pediatric Rheumatology Collaborative Study Group. N Engl J Med. 16 2000;342(11):763-769.

16. Lovell DJ, Giannini EH, Reiff A, et al. Long-term efficacy and safety of etanercept in children with polyarticular-course juvenile rheumatoid arthritis: interim results from an ongoing multicenter, open-label, extended-treatment trial. Arthritis Rheum. 2003;48(1):218-226.

17. Lovell DJ, Reiff A, Ilowite NT, et al. Safety and efficacy of up to eight years of continuous etanercept therapy in patients with juvenile rheumatoid arthritis. Arthritis Rheum. 2008;58(5):1496-1504.

18. Lovell DJ, Reiff A, Jones OY, et al. Long-term safety and efficacy of etanercept in children with polyarticular-course juvenile rheumatoid arthritis. Arthritis Rheum. 2006;54(6):1987-1994.

19. Prince FH, Twilt M, Ten Cate R, et al. Long-term follow-up on effectiveness and safety of etanercept in JIA: the Dutch national register. Ann Rheum Dis. 2008.

20. Robinson RF, Nahata MC, Hayes JR, Rennebohm R, Higgins G. Quality-of-Life Measurements in Juvenile Rheumatoid Arthritis Patients Treated with Etanercept. Clin Drug Investig. 2003;23(8):511-518.

21. Nielsen S, Ruperto N, Gerloni V, et al. Preliminary evidence that etanercept may reduce radiographic progression in juvenile idiopathic arthritis. Clin Exp Rheumatol. 2008;26(4):688-692.

22. Tynjala P, Lahdenne $P$, Vahasalo $P$, Kautiainen H, Honkanen V. Impact of anti-TNF treatment on growth in severe juvenile idiopathic arthritis. Ann Rheum Dis. 2006;65(8):1044-1049.
23. Vojvodich PF, Hansen JB, Andersson U, Savendahl L, Hagelberg S. Etanercept treatment improves longitudinal growth in prepubertal children with juvenile idiopathic arthritis. J Rheumatol. 2007;34(12):2481-2485.

24. De Benedetti F, Pignatti P, Gerloni V, et al. Differences in synovial fluid cytokine levels between juvenile and adult rheumatoid arthritis. J Rheumatol. 1997;24(7):1403-1409.

25. Jelusic M, Lukic IK, Tambic-Bukovac L, et al. Interleukin-18 as a mediator of systemic juvenile idiopathic arthritis. Clin Rheumatol. 2007;26(8):1332-1334.

26. Pascual V, Allantaz F, Arce E, Punaro M, Banchereau J. Role of interleukin-1 (IL-1) in the pathogenesis of systemic onset juvenile idiopathic arthritis and clinical response to IL-1 blockade. J Exp Med. 2005;201(9):1479-1486.

27. Yokota S, Imagawa T, Mori M, et al. Efficacy and safety of tocilizumab in patients with systemic-onset juvenile idiopathic arthritis: a randomised, double-blind, placebo-controlled, withdrawal phase III trial. Lancet. 22 2008;371(9617):998-1006.

28. Horneff G, Schmeling H, Biedermann T, et al. The German etanercept registry for treatment of juvenile idiopathic arthritis. Ann Rheum Dis. 2004;63(12):1638-1644.

29. Kimura Y, Pinho P, Walco G, et al. Etanercept treatment in patients with refractory systemic onset juvenile rheumatoid arthritis. J Rheumatol. 2005;32(5):935-942.

30. Quartier P, Taupin P, Bourdeaut F, et al. Efficacy of etanercept for the treatment of juvenile idiopathic arthritis according to the onset type. Arthritis Rheum. 2003;48(4):1093-1101.

31. Russo RA, Katsicas MM, Zelazko M. Etanercept in systemic juvenile idiopathic arthritis. Clin Exp Rheumatol. 2002;20(5):723-726.

32. Takei S, Groh D, Bernstein B, Shaham B, Gallagher K, Reiff A. Safety and efficacy of high dose etanercept in treatment of juvenile rheumatoid arthritis. J Rheumatol. 2001;28(7):1677-1680.

33. Ramanan AV, Schneider R. Macrophage activation syndrome following initiation of etanercept in a child with systemic onset juvenile rheumatoid arthritis. J Rheumatol. 2003;30(2):401-403.

34. Makay B, Yilmaz S, Turkyilmaz Z, Unal N, Oren H, Unsal E. Etanercept for therapy-resistant macrophage activation syndrome. Pediatr Blood Cancer. 2008;50(2):419-421.

35. Calin A, Dijkmans BA, Emery P, et al. Outcomes of a multicentre randomised clinical trial of etanercept to treat ankylosing spondylitis. Ann Rheum Dis. 2004;63(12):1594-1600.

36. Davis JC Jr, Van Der Heijde D, Braun J, et al. Recombinant human tumor necrosis factor receptor (etanercept) for treating ankylosing spondylitis: a randomized, controlled trial. Arthritis Rheum. 2003;48(11):3230-3236.

37. Henrickson M, Reiff A. Prolonged efficacy of etanercept in refractory enthesitis-related arthritis. J Rheumatol. 2004;31(10):2055-2061.

38. Tse SM, Laxer RM, Babyn PS, Doria AS. Radiologic Improvement of juvenile idiopathic arthritis-enthesitis-related arthritis following anti-tumor necrosis factor-alpha blockade with etanercept. J Rheumatol. 2006;33(6):1186-1188.

39. Arkela-Kautiainen M, Haapasaari J, Kautiainen H, Vilkkumaa I, Malkia E, Leirisalo-Repo M. Favourable social functioning and health related quality of life of patients with JIA in early adulthood. Ann Rheum Dis. 2005;64(6): 875-880.

40. Mease PJ, Kivitz AJ, Burch FX, et al. Etanercept treatment of psoriatic arthritis: safety, efficacy, and effect on disease progression. Arthritis Rheum. 2004;50(7):2264-2272.

41. Homeff G, Burgos-Vargas R. TNF-alpha antagonists for the treatment of juvenile-onset spondyloarthritides. Clin Exp Rheumatol. 2002; 20(6 Suppl 28):S137-S142.

42. Kotaniemi K, Kautiainen H, Karma A, Aho K. Occurrence of uveitis in recently diagnosed juvenile chronic arthritis: a prospective study. Ophthalmology. 2001;108(11):2071-2075.

43. Smith JA, Thompson DJ, Whitcup SM, et al. A randomized, placebocontrolled, double-masked clinical trial of etanercept for the treatment of uveitis associated with juvenile idiopathic arthritis. Arthritis Rheum. 2005;53(1):18-23. 
44. Schmeling $\mathrm{H}$, Horneff G. Etanercept and uveitis in patients with juvenile idiopathic arthritis. Rheumatology (Oxford). 2005;44(8):1008-1011.

45. Reiff A, Takei S, Sadeghi S, et al. Etanercept therapy in children with treatment-resistant uveitis. Arthritis Rheum. 2001;44(6):1411-1415.

46. Tynjala P, Lindahl P, Honkanen V, Lahdenne P, Kotaniemi K. Infliximab and etanercept in the treatment of chronic uveitis associated with refractory juvenile idiopathic arthritis. Ann Rheum Dis. 2007;66(4):548-550.

47. Foeldvari I, Nielsen S, Kummerle-Deschner J, et al. Tumor necrosis factor-alpha blocker in treatment of juvenile idiopathic arthritis-associated uveitis refractory to second-line agents: results of a multinational survey. J Rheumatol. 2007;34(5):1146-1150.

48. Tzaribachev N, Kuemmerle-Deschner J, Eichner M, Horneff G. Safety and efficacy of etanercept in children with juvenile idiopathic arthritis below the age of 4 years. Rheumatol Int. 2008;28(10):1031-1034.

49. Hyrich KL, Symmons DP, Watson KD, Silman AJ. Comparison of the response to infliximab or etanercept monotherapy with the response to cotherapy with methotrexate or another disease-modifying antirheumatic drug in patients with rheumatoid arthritis: results from the British Society for Rheumatology Biologics Register. Arthritis Rheum. 2006;54(6):1786-1794.

50. van der Heijde D, Klareskog L, Rodriguez-Valverde V, et al. Comparison of etanercept and methotrexate, alone and combined, in the treatment of rheumatoid arthritis: two-year clinical and radiographic results from the TEMPO study, a double-blind, randomized trial. Arthritis Rheum. 2006;54(4):1063-1074.

51. Schmeling H, Mathony K, John V, Keysser G, Burdach S, Horneff G. A combination of etanercept and methotrexate for the treatment of refractory juvenile idiopathic arthritis: a pilot study. Ann Rheum Dis. 2001;60(4):410-412.

52. Horneff G, De Bock F, Foeldvari I, et al. Safety and efficacy of combination of Etanercept and Methotrexate compared to treatment with Etanercept only in patients with juvenile idiopathic arthritis (JIA). Preliminary data from the German JIA Registry. Ann Rheum Dis. 2008 Apr 15 [Epub ahead of print].

53. Kuemmerle-Deschner JB, Horneff G. Safety and efficacy of onceweekly application of Etanercept in children with juvenile idiopathic arthritis. Rheumatol Int. 2007;28(2):153-156.

54. Prince FH, Twilt M, Jansen-Wijngaarden NC, van Suijlekom-Smit LW. Effectiveness of a once weekly double dose of etanercept in patients with juvenile idiopathic arthritis: a clinical study. Ann Rheum Dis. 2007;66(5):704-705.

55. Yim DS, Zhou H, Buckwalter M, Nestorov I, Peck CC, Lee H. Population pharmacokinetic analysis and simulation of the timeconcentration profile of etanercept in pediatric patients with juvenile rheumatoid arthritis. J Clin Pharmacol. 2005;45(3):246-256.

56. Zeltser R, Valle L, Tanck C, Holyst MM, Ritchlin C, Gaspari AA. Clinical, histological, and immunophenotypic characteristics of injection site reactions associated with etanercept: a recombinant tumor necrosis factor alpha receptor: Fc fusion protein. Arch Dermatol. 2001;137(7):893-899.

57. Winthrop KL. Risk and prevention of tuberculosis and other serious opportunistic infections associated with the inhibition of tumor necrosis factor. Nat Clin Pract Rheumatol. 2006;2(11):602-610.

58. Gerloni V, Pontikaki I, Gattinara M, Fantini F. Focus on adverse events of tumour necrosis factor alpha blockade in juvenile idiopathic arthritis in an open monocentric long-term prospective study of 163 patients. Ann Rheum Dis. 2008;67(8):1145-1152.

59. Dixon WG, Hyrich K, Watson K, Lunt M. The influence of anti-TNF therapy upon the incidence and severity of serious lower respiratory tract infections in patients with Rheumatoid Arthritis: results from the BSR biologics registry (BSRBR) Rheumatology. 2008;47(supp12):ii47.
60. Bout-Tabaku S, Rivas-Chacon R, Restrepo R. Systemic lupus erythematosus in a patient treated with etanercept for polyarticular juvenile rheumatoid arthritis. J Rheumatol. 2007;34(12):2503-2504.

61. Kanakoudi-Tsakalidou F, Tzimouli V, Pratsidou-Gertsi P, Chronopoulou E, Trachana M. The significance of persistent newly developed autoantibodies in JIA patients under long-term anti-TNF treatment. Cytokine. 2008;42(3):293-297.

62. Mohan N, Edwards ET, Cupps TR, et al. Demyelination occurring during anti-tumor necrosis factor alpha therapy for inflammatory arthritides. Arthritis Rheum. 2001;44(12):2862-2869.

63. Lepore L, Marchetti F, Facchini S, Leone V, Ventura A. Drug-induced systemic lupus erythematosus associated with etanercept therapy in a child with juvenile idiopathic arthritis. Clin Exp Rheumatol. 2003;21(2):276-277.

64. Ramos-Casals M, Brito-Zeron P, Munoz S, et al. Autoimmune diseases induced by TNF-targeted therapies: analysis of 233 cases. Medicine (Baltimore). 2007;86(4):242-251.

65. Bloom BJ. Development of diabetes mellitus during etanercept therapy in a child with systemic-onset juvenile rheumatoid arthritis. Arthritis Rheum. 2000;43(11):2606-2608.

66. van den Brande J, Hommes DW, Peppelenbosch MP. Infliximab induced T lymphocyte apoptosis in Crohn's disease. J Rheumatol Suppl. 2005;74:26-30.

67. Ruemmele FM, Prieur AM, Talbotec C, Goulet O, Schmitz J. Development of Crohn disease during anti-TNF-alpha therapy in a child with juvenile idiopathic arthritis. J Pediatr Gastroenterol Nutr. 2004;39(2):203-206.

68. Meyboom RH, Star K, Bate J, Savage R, Edwards IR. TNF-alpha inhibitors and leukaemia: international pharmacovigilance reports. Drug Saf. 2008;31(5):445-447.

69. Wolfe F, Michaud K. The effect of methotrexate and anti-tumor necrosis factor therapy on the risk of lymphoma in rheumatoid arthritis in 19,562 patients during 89,710 person-years of observation. Arthritis Rheum. 2007;56(5):1433-1439.

70. Sari I, Binicier O, Birlik M, et al. Thymic enlargement in a patient with juvenile idiopathic arthritis during etanercept therapy. Rheumatol Int. 2008.

71. Haraoui B. Differentiating the efficacy of tumor necrosis factor inhibitors. J Rheumatol Suppl. 2005;74:3-7.

72. Yilmaz M, Kendirli SG, Altintas D, Bingol G, Antmen B. Cytokine levels in serum of patients with juvenile rheumatoid arthritis. Clin Rheumatol. 2001;20(1):30-35.

73. Reiff A. The use of anakinra in juvenile arthritis. Curr Rheumatol Rep. 2005;7(6):434-440.

74. Tynjala P, Vahasalo P, Honkanen V, Lahdenne P. Drug survival of the first and second course of anti-TNF agents in juvenile idiopathic arthritis. Ann Rheum Dis. 2008 May 8 [Epub ahead of print].

75. Ruperto N, Lovell DJ, Quartier P, et al. Abatacept in children with juvenile idiopathic arthritis: a randomised, double-blind, placebocontrolled withdrawal trial. Lancet. 2008;372(9636):383-391.

76. Prince FHM, Twilt M, Simon SCM, et al. When and how to stop etanercept after successful treatment of patients with juvenile idiopathic arthritis. Pediatric Rheumatology. 2008;6 Suppl 1:P40.

77. Haapasaari J, Kautiainen H, Hannula S, Pohjankoski H, Hakala M. Good results from combining etanercept to prevailing DMARD therapy in refractory juvenile idiopathic arthritis. Clin Exp Rheumatol. 2002;20(6):867-870.

78. Kietz DA, Pepmueller PH, Moore TL. Therapeutic use of etanercept in polyarticular course juvenile idiopathic arthritis over a two year period. Ann Rheum Dis. 2002;61(2):171-173.

79. Lahdenne P, Vahasalo P, Honkanen V. Infliximab or etanercept in the treatment of children with refractory juvenile idiopathic arthritis: an open label study. Ann Rheum Dis. 2003;62(3):245-247. 
